OPEN ACCESS

Edited by:

Markus Roth,

Albert Ludwigs Universität Freiburg,

Germany

Reviewed by:

Wageesh Mishra,

Indian Institute of Astrophysics, India

Bhuwan Joshi,

Physical Research Laboratory, India

*Correspondence:

Hongqiang Song

hqsong@sdu.edu.cn

Specialty section:

This article was submitted to

Stellar and Solar Physics,

a section of the journal

Frontiers in Physics

Received: 23 March 2021

Accepted: 17 June 2021

Published: 15 July 2021

Citation:

Song H, Hu Q, Cheng X, Zhang J, Li L,

Zhao A, Wang $B$, Zheng $R$ and Chen $Y$

(2021) The Inhomogeneity of

Composition Along the Magnetic

Cloud Axis.

Front. Phys. 9:684345.

doi: 10.3389/fphy.2021.684345

\section{The Inhomogeneity of Composition Along the Magnetic Cloud Axis}

\author{
Hongqiang Song ${ }^{1,2 *}$, Qiang Hu ${ }^{3}$, Xin Cheng ${ }^{4}$, Jie Zhang ${ }^{5}$, Leping $\mathrm{Li}^{2}$, Ake Zhao ${ }^{6}$, Bing Wang ${ }^{1}$, \\ Ruisheng Zheng ${ }^{1}$ and Yao Chen ${ }^{1}$ \\ ${ }^{1}$ Shandong Provincial Key Laboratory of Optical Astronomy and Solar-Terrestrial Environment, and Institute of Space Sciences, \\ Shandong University, Weihai, China, ${ }^{2}$ CAS Key Laboratory of Solar Activity, National Astronomical Observatories, Chinese \\ Academy of Sciences, Beijing, China, ${ }^{3}$ Department of Space Science and CSPAR, University of Alabama in Huntsville, Huntsville, \\ AL, United States, ${ }^{4}$ School of Astronomy and Space Science, Nanjing University, Nanjing, China, ${ }^{5}$ Department of Physics and \\ Astronomy, George Mason University, Fairfax, VA, United States, ${ }^{6}$ College of Physics and Electric Information, Luoyang Normal \\ University, Luoyang, China
}

Coronal mass ejections (CMEs) are one of the most energetic explosions in the solar system. It is generally accepted that CMEs result from eruptions of magnetic flux ropes, which are dubbed as magnetic clouds (MCs) in interplanetary space. The composition (including the ionic charge states and elemental abundances) is determined prior to and/or during CME eruptions in the solar atmosphere and does not alter during MC propagation to $1 \mathrm{AU}$ and beyond. It has been known that the composition is not uniform within a cross section perpendicular to the MC axis, and the distribution of ionic charge states within a cross section provides us an important clue to investigate the formation and eruption processes of flux ropes due to the freeze-in effect. The flux rope is a three-dimensional magnetic structure intrinsically, and it remains unclear whether the composition is uniform along the flux rope axis as most MCs are only detected by one spacecraft. In this study, we report an MC that was observed by Advanced Composition Explorer at 1 AU during March 4-6, 1998, and Ulysses at 5.4 AU during March 24-28, 1998, sequentially. At these times, both spacecraft were located around the ecliptic plane, and the latitudinal and longitudinal separations between them were $\sim 2.2^{\circ}$ and $\sim 5.5^{\circ}$, respectively. It provides us an excellent opportunity to explore the axial inhomogeneity of flux rope composition, as both spacecraft almost intersected the cloud center at different sites along its axis. Our study shows that the average values of ionic charge states exhibit significant difference along the axis for carbon, and the differences are relatively slight but still obvious for charge states of oxygen and iron as well as the elemental abundances of iron and helium. Besides the means, the composition profiles within the cloud measured by both spacecraft also exhibit some discrepancies. We conclude that the inhomogeneity of composition exists along the cloud axis.

Keywords: coronal mass ejection, magnetic flux rope, interplanetary coronal mass ejection, magnetic cloud, ionic charge state, elemental abundance 


\section{INTRODUCTION}

Coronal mass ejections (CMEs) are an energetic explosive phenomenon in the solar atmosphere [1-4], and they are called interplanetary coronal mass ejections (ICMEs) after leaving the corona. When ICMEs interact with the Earth's magnetosphere, they can cause geomagnetic storms [5-7] and influence the normal work of high-tech equipments, such as satellites, power grids, and GPS navigation systems [8, 9]. Therefore, it is of great significance to grasp the trigger mechanisms and eruption processes of CMEs.

The researchers of the solar physics community have reached a consensus that CMEs result from eruptions of magnetic flux ropes (MFRs), which refer to a volumetric current channel with the helical magnetic field lines wrapped around the central axial field $[10,11]$. In white light coronagraph images, CMEs often exhibit a three-part structure, that is, a bright front, a dark cavity, and a bright core [12]. The cavity and core have been considered as the MFR cross section and erupted filament, respectively, for several decades. However, recent studies clearly demonstrated that both the filaments and hot channel MFRs can appear as the bright core [13-16]. The hot channels are first revealed through extreme ultraviolet passbands sensitive to high temperatures (e.g., 131 and $94 \AA$ ) [17], and they can also be observed in hard X-ray [18] and microwave [19] images. Researchers also suggest that the dark cavity corresponds to a low-density region with a sheared magnetic field in the early eruption stage [16].

Both theoretical and observational studies reveal that MFRs can form prior to [17, 20-23] and during [24-28] solar eruptions, while they might exist before eruptions in more events [29]. The numerical simulations demonstrate that the repetitive magnetic reconnections could play an important role during the MFR evolution [30]. The remote-sensing observations have been widely used to investigate the MFR formation process $[26,31$, 32]. The charge states within ICMEs are frozen-in near the Sun [33], and the relative abundances of elements with different first ionization potentials (FIPs) are different obviously in the corona and photosphere $[34,35]$. As the composition does not alter during CME propagation to $1 \mathrm{AU}$ and beyond [36], the in situ data are also employed to analyze the MFR formation [28, 37, 38] and plasma origin $[39,40]$ of CMEs. So far, the most complete composition data of ICMEs are provided by the solar wind ion composition spectrometer (SWICS) aboard Advanced Composition Explorer (ACE) and Ulysses, which can provide the charge states and elemental abundances of $\sim 10$ elements [41].

When an ICME has its nose pass through a spacecraft, the MFR will be detected as a magnetic cloud (MC) [42-44]. This is schematically shown in Figure 1A (also see $[45,46]$ for a similar cartoon), where the purple arrow depicts a spacecraft trajectory crossing one ICME through its nose portion as marked with the blue rectangle. Figure 1B displays the MFR within the rectangle, and the green dots represent the center of each cross section. The black, blue, and red arrows depict three different trajectories.

Several statistical studies have been conducted on ICME composition. Huang et al. [47] analyzed the composition inside $124 \mathrm{MCs}$ and reported that fast MCs have higher charge states and relative elemental abundances (except the

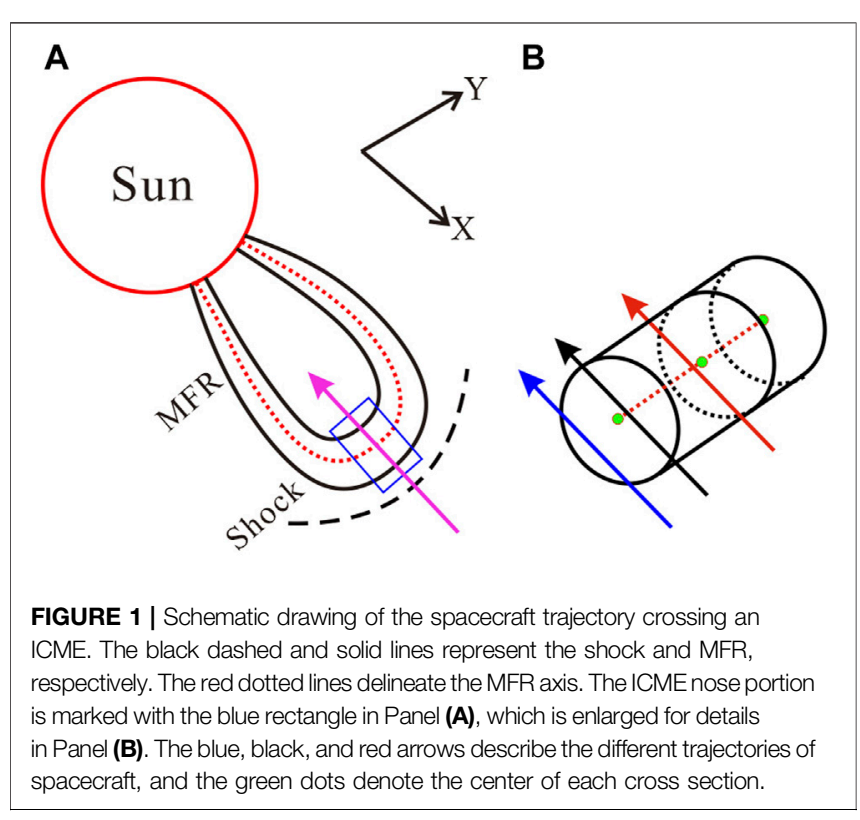

$\mathrm{C} / \mathrm{O})$ than slow ones. Owens [48] analyzed the charge states of carbon, oxygen, and iron within 215 ICMEs, including $97 \mathrm{MCs}$ and 118 non-cloud events, and found that MCs exhibit higher ionic charge states than non-cloud events. Zurbuchen et al. [49] performed a comprehensive analysis of the elemental abundances of 310 ICMEs from 1998 March to 2011 August. They reported that the abundances of low-FIP elements within ICMEs exhibit a systematic increase compared to the solar wind, and the ICMEs with elevated iron charge states possess higher FIP fractionation than the other ICMEs. Very recently, Song et al. [50] reported that all the ICME compositions possess the solar cycle dependence.

In the meantime, some attentions are paid on the composition distribution inside each MC. Song et al. [37] found that the average values of iron charge states $\left(\left\langle\mathrm{Q}_{\mathrm{Fe}}\right\rangle\right)$ can present four regular profiles along the spacecraft trajectories throughout MCs, that is, (i) a bimodal profile with both peaks higher than $12+$, (ii) a unimodal profile with peaks higher than $12+$, and (iii) and (iv) the $\left.<\mathrm{Q}_{\mathrm{Fe}}\right\rangle$ profile remains beyond and below $12+$ throughout the spacecraft trajectory inside an MC, respectively. Their studies demonstrated that the charge states can be non-uniform within the cross section of a specified MC and suggested that the above profiles are tightly correlated with both the impact factor of spacecraft trajectories and the formation process of MFRs. For example, the bimodal profile implies that the MFR exists prior to eruption; see Figure 8 in [37] for more details. In addition, the elemental abundances are not uniform within one cross section either [39]. Therefore, a spacecraft can detect different composition profiles when it crosses one MC along the blue and black arrows as shown in Figure 1B, which are located in the same cross section perpendicular to the axis but with different impact factors. However, whether the inhomogeneity of composition exists along the $\mathrm{MC}$ axis remains unclear because most MCs are detected only by either ACE or Ulysses. Given the $\mathrm{MC}$ is a three-dimensional (3D) structure intrinsically, the axial distribution of composition can reveal whether different portions 
along the MFR axis experience different eruption processes in the corona.

In this study, we report an intriguing event, in which an MC was observed by ACE at 1 AU during March 4-6, 1998, and Ulysses at $~ 5.4$ AU during March 24-28, 1998. At these times, both spacecraft were located around the ecliptic plane, and the latitudinal and longitudinal separations between them were $\sim 2.2^{\circ}$ and $\sim 5.5^{\circ}$, respectively. The Grad-Shafranov (GS) reconstruction $[51,52]$ demonstrated that the $\mathrm{MC}$ axis oriented in an approximate east-west direction with the axis direction at Ulysses being tilted slightly away from that at ACE, and both spacecraft almost intersected the MC center [53]. This implies that the two spacecraft cross the $\mathrm{MC}$ along two trajectories resembling the black and red arrows in Figure 1B, respectively, and provide us an excellent opportunity to explore whether the composition is uniform along the axis. We introduce the data in Section 2 and give the observations in Section 3. Section 4 presents the conclusion and discussion.

\section{DATA}

The data used in this study are provided by several payloads on board the ACE and Ulysses spacecraft. ACE is in a halo orbit around the first Lagrangian point between the Earth and the Sun since it was launched in 1997. Ulysses was launched in 1990 and entered an elliptical and heliocentric orbit with an aphelion at 5.4 AU from the Sun and a perihelion distance of $\sim 1.34 \mathrm{AU}$. Magnetic field data are provided by ACE/MAG [54] and Ulysses/ magnetic field [55] instruments. The bulk solar wind properties and the helium abundances are from the Solar Wind Electron, Proton, and Alpha Monitor (SWEPAM) [56] on board ACE and the Solar Wind Observations Over the Poles of the Sun (SWOOPS) [57] on board Ulysses. The SWICS instruments on board both spacecraft $[58,59]$ offer the composition of heavy ions.

\section{OBSERVATIONS}

The criteria used to identify MCs near 1 AU mainly include the enhanced magnetic field strength, smoothly changing of magnetic field direction, declining profile of solar wind velocity, low proton temperature (or low plasma $\beta$ ), and elevated $\mathrm{He}^{2+} / \mathrm{H}^{+}$ratio $[42,60,61]$. ACE detected an MC during March 4-6, 1998, as shown in Figure 2. The vertical dashed line denotes the shock driven by the ICME, and the two dash-dotted lines demarcate the MC boundaries.

Figure 2A shows the total magnetic field strength and its three components in RTN coordinate, where the $\mathrm{x}$-axis (R) points from Sun center to spacecraft, the $\mathrm{y}$-axis $(\mathrm{T})$ is the cross product of solar rotational axis and $\mathrm{X}$ axis, lying in the solar equatorial plane towards the west limb, and the $\mathrm{z}$-axis $(\mathrm{N})$ is the cross product of $\mathrm{x}$ and $y$ axes. The total magnetic field strength (black) increased obviously compared to the background solar wind, and the Bn component (blue) changed its direction gradually within the $\mathrm{MC}$, which are the typical features of MCs. Figures 2B-D present the

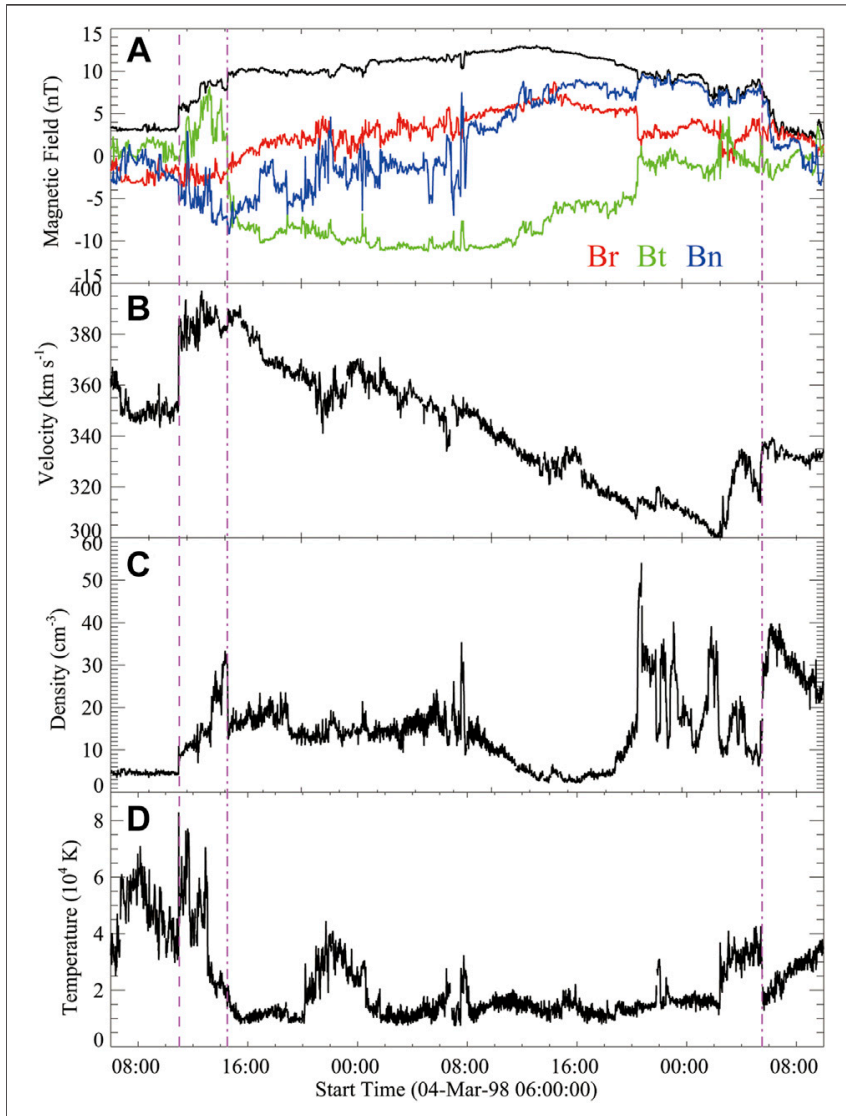

FIGURE 2 | Magnetic field and solar wind parameters measured by ACE near $1 \mathrm{AU}$. (A) Total magnetic field strength (black) and its three components in RTN coordinate and (B-D) velocity, density, and temperature of solar wind. The vertical dashed line denote the shock, and the dash-dotted lines demarcate the MC boundaries.

velocity, density, and temperature of the ICME sequentially. The declining profile of velocity indicates that the MFR is expanding.

Ulysses detected an MC during March 24-28 [62] as shown in Figure 3, where the magnetic field, velocity, density, and temperature are presented from top to bottom panels sequentially. The velocity profile in Figure $3 \mathbf{B}$ shows that the MC keeps expansion during the propagation to 5.4 AU. Due to the continuous expansion, the total magnetic field intensity within this $\mathrm{MC}$ decreased obviously near 5.4 AU compared to $\sim 1 \mathrm{AU}$, see Figures 2A, 3A. A shock exists within the MC as depicted with the red arrows in Figures $\mathbf{3 A}, \mathbf{B}$, and the $\mathrm{MC}$ rear boundary can be identified through the $\mathrm{He}^{2+} / \mathrm{H}^{+}$ratio and the plasma $\beta$ value [53]. Note that the shock does not influence our analyses about the ionic charge states and elemental abundances.

Previous studies $[53,63]$ have confirmed that the MC displayed in Figure 3 corresponds to that in Figure 2. Skoug et al. [63] fitted both MCs using a force-free model of the magnetic field [64] and found that their central speed and cloud axis direction were very similar. The increase in MC diameter between 1 and 5.4 AU was also consistent with an expanding MC. Besides, both MCs had left-handed field structure and contained the similar magnetic fluxes, which 


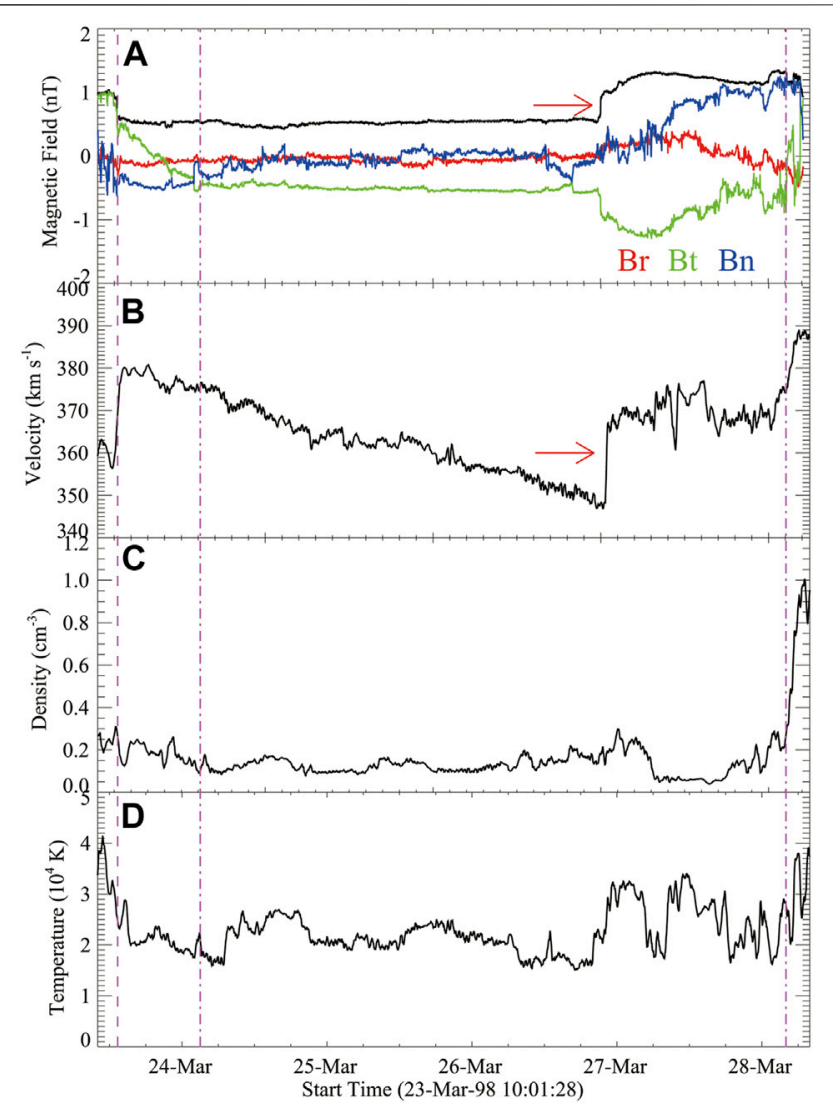

FIGURE 3 | Magnetic field and solar wind parameters measured by Ulysses near 5.4 AU. (A) Total magnetic field strength (black) and its three components in RTN coordinate and (B-D) velocity, density, and temperature of solar wind. The vertical dashed line denotes the shock, and the dashdotted lines demarcate the MC boundaries. The red arrows in (A) and (B) depict the shock inside the MC.

were further confirmed by $\mathrm{Du}$ et al. [53] with the GS reconstruction technique. In addition, $\mathrm{Du}$ et al. [53] input the plasma and magnetic field data observed by ACE to their magnetohydrodynamic model to simulate the MC propagation and evolution to the Ulysses location. They compared the model predictions and the Ulysses observations, and identified further that Ulysses and ACE observed the same MC. As mentioned, the ACE (at 1 AU) and Ulysses (at $~ 5.4 \mathrm{AU}$ ) were located near the ecliptic plane with a latitudinal separation of $\sim 2.2^{\circ}$ and a longitudinal separation of $\sim 5.5^{\circ}$ when they detected the MC. The GS reconstruction showed that the MC axis oriented in an approximate east-west direction, and both spacecraft almost intersected the MC center [53], which support that ACE and Ulysses crossed the $\mathrm{MC}$ at different sites along its axis and provide us an excellent opportunity to explore whether the axial composition is uniform.

We compare the composition measured by both spacecraft in Figure 4, where the black and red lines represent the results of ACE and Ulysses, respectively. Please note that we only plot the composition within the MC, that is, the left/right boundary of each panel corresponds to the MC start/end time. The ionic

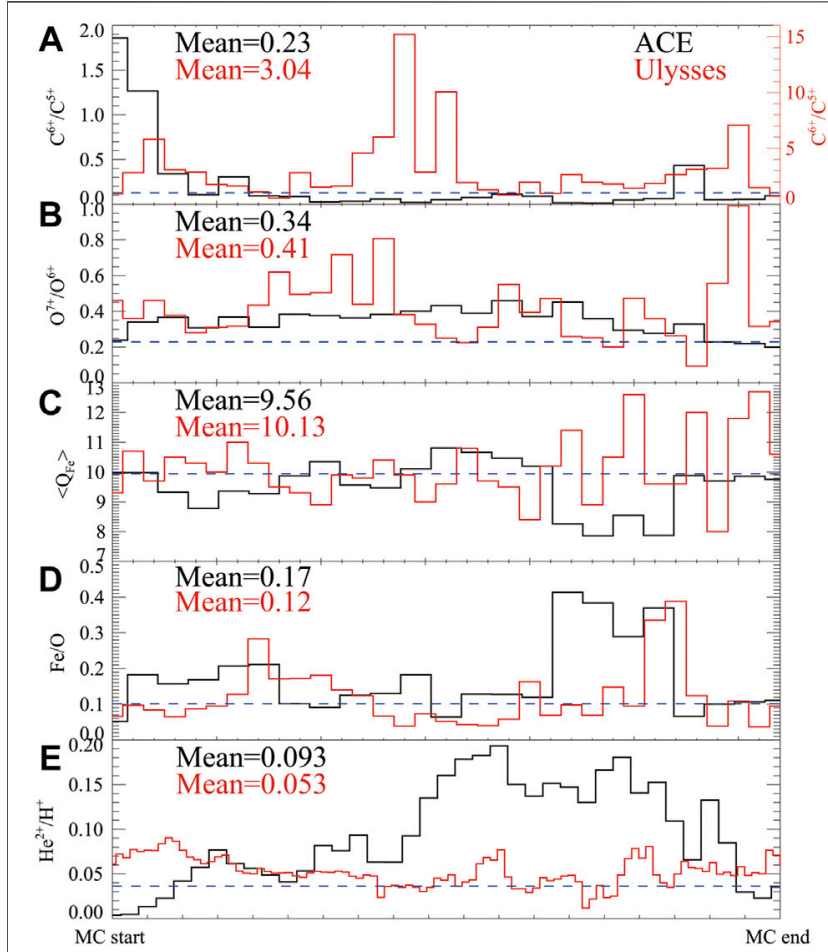

FIGURE 4 | Composition within the MC provided by SWICS aboard ACE (black) and Ulysses (red). Panels (A-E) show the $\mathrm{C}^{6+} / \mathrm{C}^{5+}, \mathrm{O}^{7+} / \mathrm{O}^{6+},<\mathrm{Q}_{\mathrm{Fe}}>$, $\mathrm{Fe} / \mathrm{O}$, and $\mathrm{He}^{2+} / \mathrm{H}^{+}$sequentially, and their average values are also presented in each panel. Note that the Ulysses values in Panel (A) correspond to the right ordinate. The blue horizontal dashed lines depict the corresponding means of slow wind during solar maximum [65]. The MC started from 14:30 UT on March 4 (3:00 UT on March 24) and ended at 5:30 UT on March 6 (4:00 UT on March 28) for ACE (Ulysses).

charge states $\left(\mathrm{C}^{6+} / \mathrm{C}^{5+}, \mathrm{O}^{7+} / \mathrm{O}^{6+}\right.$, and $\left\langle\mathrm{Q}_{\mathrm{Fe}}\right\rangle$ and elemental abundances $\left(\mathrm{Fe} / \mathrm{O}\right.$ and $\left.\mathrm{He}^{2+} / \mathrm{H}^{+}\right)$are presented in Figure 4A-E. The average values within the $\mathrm{MC}$ are also shown in each panel. The blue horizontal dashed lines represent the corresponding means in the slow solar wind during solar maximum [65] for reference and comparison.

Our study shows that the average values of composition within an $\mathrm{MC}$ can possess significant differences along the axis. For example, the $\mathrm{C}^{6+} / \mathrm{C}^{5+}$ ratio measured by Ulysses (3.04) is 12 times higher than that by ACE (0.23). In the meantime, the differences could be relatively slight for some compositions. For example, the $\mathrm{O}^{7+} / \mathrm{O}^{6+}$, ratio measured by Ulysses $(0.41)$ is higher than that of ACE (0.34) by $\sim 21 \%$. The means of $\left\langle\mathrm{Q}_{\mathrm{Fe}}\right\rangle$ detected by both spacecraft are nearly identical $(\sim 10)$. As to the elemental abundance, the $\mathrm{Fe} / \mathrm{O}$ ratio by $\mathrm{ACE}(0.17)$ is $\sim 42 \%$ higher than that by Ulysses $(0.12)$, and the $\mathrm{He}^{2+} / \mathrm{H}^{+}$ratio of ACE $(0.093)$ is higher than that of Ulysses $(0.053)$ by $\sim 75 \%$.

Besides the average values, the composition profiles measured by both spacecraft also exhibit discrepancy. Figure 4A shows that the $\mathrm{C}^{6+} / \mathrm{C}^{5+}$ of Ulysses elevated at the MC center, while the ACE profile did not exhibit the central peak. The $\mathrm{O}^{7+} / \mathrm{O}^{6+}$ of Ulysses presented a multi-peak profile, while ACE did not detect obvious peaks as shown in Figure 4B. The $\mathrm{He}^{2+} / \mathrm{H}^{+}$of ACE elevated in the 
second half as displayed in Figure 4E, different from the profile of Ulysses that did not have large variation along the whole path. These can rule out the possibility that the inhomogeneity of composition is induced by the erosion [66] completely during propagation from 1 to $5.4 \mathrm{AU}$. Moreover, the erosion effect should be small for this event as both MCs have the similar magnetic fluxes as mentioned. The profiles of $\left\langle\mathrm{Q}_{\mathrm{Fe}}\right\rangle$ and $\mathrm{Fe} / \mathrm{O}$ measured by both spacecraft also exhibit some different fluctuation characteristics as displayed in Figures 4C,D. The above results prove that the composition is inhomogeneous along the MC axis.

\section{CONCLUSION AND DISCUSSION}

An MC was detected by ACE at $~ 1 \mathrm{AU}$ and Ulysses at $~ 5.4 \mathrm{AU}$ sequentially during March 1998, when both spacecraft were located around the ecliptic plane. The latitudinal and longitudinal separations between them were $\sim 2.2^{\circ}$ and $\sim 5.5^{\circ}$, respectively. The GS reconstruction [53] showed that the axis oriented in an approximate east-west direction, and both spacecraft almost intersected the MC center, which provided an excellent opportunity to explore whether the composition is uniform along the axis. We compared the ionic charge states of carbon, oxygen, and iron $\left(\mathrm{C}^{6+} / \mathrm{C}^{5+}, \mathrm{O}^{7+} / \mathrm{O}^{6+}\right.$, and $\left.<\mathrm{Q}_{\mathrm{Fe}}>\right)$, as well as the elemental abundances of iron and helium ( $\mathrm{Fe} / \mathrm{O}$ and $\mathrm{He}^{2+}$ / $\mathrm{H}^{+}$) along the two trajectories. The results showed that the average values of $\mathrm{C}^{6+} / \mathrm{C}^{5+}$ exhibit significant difference along the axis, while the differences are relatively slight but still obvious for $\mathrm{O}^{7+} / \mathrm{O}^{6+},<\mathrm{Q}_{\mathrm{Fe}}>, \mathrm{Fe} / \mathrm{O}$, and $\mathrm{He}^{2+} / \mathrm{H}^{+}$. Besides the means, the composition profiles within the $\mathrm{MC}$ measured by both spacecraft also exhibit obvious discrepancies. We conclude that the inhomogeneity of composition exists along the MC axis.

The magnetic field within the MC measured by Ulysses did not exhibit the obvious changing of direction compared with the measurements of ACE, see Figures 2A, 3A. This might indicate that Ulysses passed through the ICME along a path a little far from the $\mathrm{MC}$ center than ACE. Figure 4A showed that Ulysses detected high $\mathrm{C}^{6+} / \mathrm{C}^{5+}$ at its central portion, which should also be observed by $\mathrm{ACE}$ if the composition is uniform along the MC axis. However, the $\mathrm{C}^{6+} / \mathrm{C}^{5+}$ profile of ACE did not present the elevated center. Therefore, if assuming there were some uncertainties about the spacecraft path in the GS reconstruction, it will not change our conclusion about the axial inhomogeneity of MC composition.

The charge states of carbon, oxygen, and iron are frozen-in sequentially in the corona, that is, the frozen-in altitudes of carbon and iron are the lowest and highest, respectively, in these three elements. For example, carbon is frozen-in below 1.5 solar radii $[67,68]$, while the iron around three to four solar radii $[69,70]$. Therefore, the obvious differences of $\mathrm{C}^{6+} / \mathrm{C}^{5+}$ along the MC axis imply that the different portions of MFR along the axis experience eruption processes with different physical parameters (e.g., temperature, density, and velocity) in the low corona. The similar values of $\left\langle\mathrm{Q}_{\mathrm{Fe}}\right\rangle$ indicate that the physical parameters along the axis approached in the high corona. These should be taken into account in $3 \mathrm{D}$ simulations of CMEs. The axial inhomogeneity of elemental abundances implies that the abundances are not uniform throughout the MC source region on the Sun.

Our study demonstrated that the axial composition is nonuniform inside an MC, while we cannot conclude that this large inhomogeneity exists within each MC. More events are necessary to investigate the inhomogeneity of composition along the MC axis, which needs a CME being detected by several spacecraft sequentially or simultaneously at different locations. This becomes more realizable as Solar Orbiter was launched in 2020 [71]. Besides, Chinese solar physicists are proposing several space missions [72] to explore the Sun and solar eruption further. The Lay a Finger on the Sun [73] will launch a spacecraft to explore the solar eruption near the Sun; thus, it will provide more MC cases that are measured sequentially near the Sun and around $1 \mathrm{AU}$ combined with other spacecraft. The Solar Ring [74] plans to deploy six spacecraft, grouped in three pairs, on a sub-AU orbit around the Sun. The two spacecraft in each group are separated by $\sim 30^{\circ}$ and every two groups by $\sim 120^{\circ}$, which can provide more cases that are measured simultaneously by two or more spacecraft around the ecliptic plane. All of these missions will facilitate the studies of solar eruptions and other related issues.

\section{AUTHOR CONTRIBUTIONS}

HS led the analysis and drafted the manuscript. QH contributed to further understand the GS reconstruction results in [53]. XC, JZ, LL, and $\mathrm{AZ}$ provided suggestions to improve the research. BW helped to plot Figure 1. RZ and YC contributed to improve the manuscript.

\section{DATA AVAILABILITY STATEMENT}

The plasma and magnetic field data of both ACE and Ulysses are publicly available at the ACE science center (http://www.srl. caltech.edu/ACE/ASC/level2/index.html) and the Ulysses Final Archive (http://ufa.esac.esa.int/ufa/), respectively.

\section{FUNDING}

This work was supported by CAS grants XDA-17040507 and NSFC grants U2031109, 11790303 (No. 11790300), and No. 12073042. HS was also supported by the open research program of the CAS Key Laboratory of Solar Activity, KLSA202107.

\section{ACKNOWLEDGMENTS}

The authors thank the referees for their constructive comments and suggestions which helped improve the original manuscript considerably. The authors acknowledge the use of data provided by ACE and Ulysses missions. HS thanks Dr Ying D. Liu for the helpful discussions. 


\section{REFERENCES}

1. Chen PF. Coronal Mass Ejections: Models and Their Observational Basis. Living Rev Solar Phys (2011) 8:1. doi:10.12942/lrsp-2011-1

2. Webb DF, and Howard TA. Coronal Mass Ejections: Observations. Living Rev Solar Phys (2012) 9:3. doi:10.12942/lrsp-2012-3

3. Cheng X, Guo Y, and Ding M. Origin and Structures of Solar Eruptions I: Magnetic Flux Rope. Sci China Earth Sci (2017) 60:1383-407. doi:10.1007/ s11430-017-9074-6

4. Guo Y, Cheng X, and Ding M. Origin and Structures of Solar Eruptions II: Magnetic Modeling. Sci China Earth Sci (2017) 60:1408-39. doi:10.1007/ s11430-017-9081-x

5. Gosling JT, McComas DJ, Phillips JL, and Bame SJ. Geomagnetic Activity Associated with Earth Passage of Interplanetary Shock Disturbances and Coronal Mass Ejections. J Geophys Res (1991) 96:7831-9. doi:10.1029/ 91JA00316

6. Zhang J, Richardson IG, Webb DF, Gopalswamy N, Huttunen E, and Kasper JC. Solar and Interplanetary Sources of Major Geomagnetic Storms (Dst <= -100 nT) during 1996-2005. J Geophys Res (Space Physics) (2007) 112:A10102. doi:10.1029/2007JA012321

7. Xu M, Shen C, Wang Y, Luo B, and Chi Y. Importance of Shock Compression in Enhancing ICME's Geoeffectiveness. ApJ (2019) 884:L30. doi:10.3847/20418213/ab4717

8. Cannon PS. Extreme Space Weather-A Report Published by the UK Royal Academy of Engineering. Space Weather (2013) 11:138-9. doi:10.1002/ swe.20032

9. Riley P, Baker D, Liu YD, Verronen P, Singer H, and Güdel M. Extreme Space Weather Events: From Cradle to Grave. Space Sci Rev (2018) 214:21. doi:10.1007/s11214-017-0456-3

10. Gibson SE, and Fan Y. Coronal Prominence Structure and Dynamics: A Magnetic Flux Rope Interpretation. J Geophys Res (2006) 111:A12103. doi:10.1029/2006JA011871

11. Canou A, and Amari T. A Twisted Flux Rope as the Magnetic Structure of a Filament in Active Region 10953 Observed Byhinode. ApJ (2010) 715:1566-74. doi:10.1088/0004-637X/715/2/1566

12. Illing RME, and Hundhausen AJ. Observation of a Coronal Transient from 1.2 to 6 Solar Radii. J Geophys Res (1985) 90:275-82. doi:10.1029/ JA090iA01p00275

13. Howard TA, DeForest CE, Schneck UG, and Alden CR. Challenging Some Contemporary Views of Coronal Mass Ejections. II. The Case for Absent Filaments. ApJ (2017) 834:86. doi:10.3847/1538-4357/834/1/86

14. Song HQ, Cheng X, Chen Y, Zhang J, Wang B, Li LP, et al. The Three-Part Structure of a Filament-Unrelated Solar Coronal Mass Ejection. ApJ (2017) 848:21. doi:10.3847/1538-4357/aa8d1a

15. Song HQ, Zhang J, Cheng X, Li LP, Tang YZ, Wang B, et al. On the Nature of the Bright Core of Solar Coronal Mass Ejections. ApJ (2019) 883:43. doi:10.3847/1538-4357/ab304c

16. Song HQ, Zhang J, Li LP, Liu YD, Zhu B, Wang B, et al. The Structure of Solar Coronal Mass Ejections in the Extreme-Ultraviolet Passbands. ApJ (2019) 887: 124. doi:10.3847/1538-4357/ab50b6

17. Zhang J, Cheng X, and Ding M-d. Observation of an Evolving Magnetic Flux Rope before and during a Solar Eruption. Nat Commun (2012) 3:747. doi:10.1038/ncomms1753

18. Sahu S, Joshi B, Mitra PK, Veronig AM, and Yurchyshyn V. Hard X-Ray Emission from an Activated Flux Rope and Subsequent Evolution of an Eruptive Long-Duration Solar Flare. ApJ (2020) 897:157. doi:10.3847/15384357/ab962b

19. Wu Z, Chen Y, Huang G, Nakajima H, Song H, Melnikov V, et al. Microwave Imaging of a Hot Flux Rope Structure during the Pre-impulsive Stage of an Eruptive M7.7 Solar Flare. ApJ (2016) 820:L29. doi:10.3847/2041-8205/820/ $2 /$ L29

20. Chen J. Theory of Prominence Eruption and Propagation: Interplanetary Consequences. J Geophys Res (1996) 101:27499-519. doi:10.1029/96JA02644

21. Lin J, and Forbes TG. Effects of Reconnection on the Coronal Mass Ejection Process. J Geophys Res (2000) 105:2375-92. doi:10.1029/1999JA900477

22. Patsourakos S, Vourlidas A, and Stenborg G. Direct Evidence for a Fast Coronal Mass Ejection Driven by the Prior Formation and Subsequent
Destabilization of a Magnetic Flux Rope. ApJ (2013) 764:125. doi:10.1088/ 0004-637X/764/2/125

23. Cheng X, Zhang J, Ding MD, Liu Y, and Poomvises W. The Driver of Coronal Mass Ejections in the Low Corona: A Flux Rope. ApJ (2013) 763:43. doi:10.1088/0004-637X/763/1/43

24. Mikic Z, and Linker JA. Disruption of Coronal Magnetic Field Arcades. ApJ (1994) 430:898. doi:10.1086/174460

25. Antiochos SK, DeVore CR, and Klimchuk JA. A Model for Solar Coronal Mass Ejections. ApJ (1999) 510:485-93. doi:10.1086/306563

26. Song HQ, Zhang J, Chen Y, and Cheng X. Direct Observations of Magnetic Flux Rope Formation during a Solar Coronal Mass Ejection. ApJ (2014) 792: L40. doi:10.1088/2041-8205/792/2/L40

27. Ouyang Y, Yang K, and Chen PF. Is Flux Rope a Necessary Condition for the Progenitor of Coronal Mass Ejections?. Astrophys J (2015) 815:72. doi:10.1088/ 0004-637X/815/1/72

28. Wang W, Liu R, Wang Y, Hu Q, Shen C, Jiang C, et al. Buildup of a Highly Twisted Magnetic Flux Rope during a Solar Eruption. Nat Commun (2017) 8: 1330. doi:10.1038/s41467-017-01207-x

29. Ouyang Y, Zhou YH, Chen PF, and Fang C. Chirality and Magnetic Configurations of Solar Filaments. Astrophys J (2017) 835:94. doi:10.3847/ $1538-4357 / 835 / 1 / 94$

30. Kumar S, Bhattacharyya R, Joshi B, and Smolarkiewicz PK. On the Role of Repetitive Magnetic Reconnections in Evolution of Magnetic Flux Ropes in Solar Corona. ApJ (2016) 830:80. doi:10.3847/0004-637X/830/2/80

31. Cheng $X$, Ding MD, and Fang C. Imaging and Spectroscopic Diagnostics on the Formation of Two Magnetic Flux Ropes Revealed Bysdo/aia Andiris. ApJ (2015) 804:82. doi:10.1088/0004-637X/804/2/82

32. Zheng R, Chen $\mathrm{Y}$, Wang B, Song H, and Cao W. Formation of a Tiny Flux Rope in the center of an Active Region Driven by Magnetic Flux Emergence, Convergence, and Cancellation. A\&A (2020) 642:A199. doi:10.1051/0004$6361 / 202037475$

33. Owocki SP, Holzer TE, and Hundhausen AJ. The Solar Wind Ionization State as a Coronal Temperature Diagnostic. ApJ (1983) 275:354-66. doi:10.1086/161538

34. Laming JM. The FIP and Inverse FIP Effects in Solar and Stellar Coronae. Living Rev Sol Phys (2015) 12:2. doi:10.1007/lrsp-2015-2

35. Vadawale SV, Mondal B, Mithun NPS, Sarkar A, Janardhan P, Joshi B, et al. Observations of the Quiet Sun during the Deepest Solar Minimum of the Past Century with Chandrayaan-2 XSM: Elemental Abundances in the Quiescent Corona. ApJL (2021) 912:L12. doi:10.3847/2041-8213/abf35d

36. Song $\mathrm{H}$, and Yao S. Characteristics and Applications of Interplanetary Coronal Mass Ejection Composition. Sci China Technol Sci (2020) 63:2171. doi:10.1007/s11431-020-1680-y

37. Song HQ, Zhong Z, Chen Y, Zhang J, Cheng X, Zhao L, et al. A Statistical Study of the Average Iron Charge State Distributions inside Magnetic Clouds for Solar Cycle 23. ApJS (2016) 224:27. doi:10.3847/0067-0049/224/2/27

38. Huang J, Liu YC-M, Peng J, Qi Z, Li H, Klecker B, et al. The Distributions of Iron Average Charge States in Small Flux Ropes in Interplanetary Space: Clues to Their Twisted Structures. J Geophys Res Space Phys (2018) 123:7167-80. doi:10.1029/2018JA025660

39. Song HQ, Chen Y, Li B, Li LP, Zhao L, He JS, et al. The Origin of Solar Filament Plasma Inferred from In Situ Observations of Elemental Abundances. ApJ (2017) 836:L11. doi:10.3847/2041-8213/aa5d54

40. Fu H, Harrison RA, Davies JA, Xia L, Zhu X, Li B, et al. The High Helium Abundance and Charge States of the Interplanetary CME and its Material Source on the Sun. ApJ (2020) 900:L18. doi:10.3847/2041-8213/abb083

41. Lepri ST, Zurbuchen TH, Fisk LA, Richardson IG, Cane HV, and Gloeckler G. Iron Charge Distribution as an Identifier of Interplanetary Coronal Mass Ejections. J Geophys Res (2001) 106:29231-8. doi:10.1029/2001JA000014

42. Burlaga L, Sittler E, Mariani F, and Schwenn R. Magnetic Loop behind an Interplanetary Shock: Voyager, Helios, and IMP 8 Observations. J Geophys Res (1981) 86:6673-84. doi:10.1029/JA086iA08p06673

43. Syed Ibrahim M, Joshi B, Cho K-S, Kim R-S, and Moon Y-J. Interplanetary Coronal Mass Ejections during Solar Cycles 23 and 24: Sun-Earth Propagation Characteristics and Consequences at the Near-Earth Region. Sol Phys (2019) 294:54. doi:10.1007/s11207-019-1443-5

44. Song HQ, Zhang J, Cheng X, Li G, Hu Q, Li LP, et al. Do All Interplanetary Coronal Mass Ejections Have a Magnetic Flux Rope Structure Near 1 au?. ApJ (2020) 901:L21. doi:10.3847/2041-8213/abb6ec 
45. Gopalswamy N. Properties of Interplanetary Coronal Mass Ejections. Space Sci Rev (2007) 124:145-68. doi:10.1007/s11214-006-9102-1

46. Kim R-S, Gopalswamy N, Cho K-S, Moon Y-J, and Yashiro S. Propagation Characteristics of CMEs Associated with Magnetic Clouds and Ejecta. Sol Phys (2013) 284:77-88. doi:10.1007/s11207-013-0230-y

47. Huang J, Liu Y, Feng H, Zhao A, Abidin ZZ, Shen Y, et al. A Statistical Study of the Plasma and Composition Distribution inside Magnetic Clouds: 1998-2011. ApJ (2020) 893:136. doi:10.3847/1538-4357/ab7a28

48. Owens MJ. Solar Wind and Heavy Ion Properties of Interplanetary Coronal Mass Ejections. Sol Phys (2018) 293:122. doi:10.1007/s11207-018-1343-0

49. Zurbuchen TH, Weberg M, von Steiger R, Mewaldt RA, Lepri ST, and Antiochos SK. Composition of Coronal Mass Ejections. ApJ (2016) 826:10. doi:10.3847/0004-637X/826/1/10

50. Song H, Li L, Sun Y, Lv Q, Zheng R, and Chen Y. Solar Cycle Dependence of ICME Composition. arXiv:2106.03003 (2021).

51. Hu Q, and Sonnerup BUÖ. Reconstruction of Magnetic Clouds in the Solar Wind: Orientations and Configurations. J Geophys Res (2002) 107:1142. doi:10.1029/2001JA000293

52. Hu Q. The Grad-Shafranov Reconstruction in Twenty Years: 1996-2016. Sci China Earth Sci (2017) 60:1466-94. doi:10.1007/s11430-016-9052-110.1007/ s11430-017-9067-2

53. Du D, Wang C, and Hu Q. Propagation and Evolution of a Magnetic Cloud from ACE to Ulysses. J Geophys Res (2007) 112:a. doi:10.1029/2007JA012482

54. Smith CW, L'Heureux J, Ness NF, Acuña MH, Burlaga LF, and Scheifele J. The ACE Magnetic Fields Experiment. Space Sci Rev (1998) 86:613-32. doi:10.1023/A:100509221666810.1007/978-94-011-4762-0_21

55. Balogh A, Beek TJ, Forsyth RJ, Hedgecock PC, Marquedant RJ, Smith EJ, et al. The Magnetic Field Investigation on the ULYSSES mission Instrumentation and Preliminary Scientific Results. Astron Astrophys Suppl (1992) 92:221-36.

56. McComas DJ, Bame SJ, Barker P, Feldman WC, Phillips JL, Riley P, et al. Solar Wind Electron Proton Alpha Monitor (SWEPAM) for the Advanced Composition Explorer. Space Sci Rev (1998) 86:563-612. doi:10.1023/A: 100504023259710.1007/978-94-011-4762-0_20

57. Bame SJ, McComas DJ, Barraclough BL, Phillips JL, Sofaly KJ, Chavez JC, et al. The ULYSSES Solar Wind Plasma experiment. Astron Astrophys Suppl (1992) 92:237-65.

58. Gloeckler G, Cain J, Ipavich FM, Tums EO, Bedini P, Fisk LA, et al. Investigation of the Composition of Solar and Interstellar Matter Using Solar Wind and Pickup Ion Measurements with SWICS and SWIMS on the ACE Spacecraft. Space Sci Rev (1998) 86:497-539. doi:10.1023/A:100503613168910.1007/978-94-011-4762-0_18

59. Geiss J, Gloeckler G, Balsiger H, Fisk LA, Galvin AB, Gliem F, et al. Plasma Composition in Jupiter's Magnetosphere: Initial Results from the Solar Wind Ion Composition Spectrometer. Science (1992) 257:1535-9. doi:10.1126/ science.257.5076.1535

60. Cane HV, and Richardson IG. Interplanetary Coronal Mass Ejections in the Near-Earth Solar Wind during 1996-2002. J Geophys Res (2003) 108:1156. doi:10.1029/2002JA009817

61. Chi Y, Shen C, Wang Y, Xu M, Ye P, and Wang S. Statistical Study of the Interplanetary Coronal Mass Ejections from 1995 to 2015. Sol Phys (2016) 291: 2419-39. doi:10.1007/s11207-016-0971-5
62. Du D, Zuo PB, and Zhang XX. Interplanetary Coronal Mass Ejections Observed by Ulysses through its Three Solar Orbits. Sol Phys (2010) 262: 171-90. doi:10.1007/s11207-009-9505-8

63. Skoug RM, Feldman WC, Gosling JT, McComas DJ, Reisenfeld DB, Smith CW, et al. Radial Variation of Solar Wind Electrons inside a Magnetic Cloud Observed at 1 and 5 AU. J Geophys Res (2000) 105:27269-75. doi:10.1029/2000JA000095

64. Lepping RP, Jones JA, and Burlaga LF. Magnetic Field Structure of Interplanetary Magnetic Clouds at 1 AU. J Geophys Res (1990) 95: 11957-65. doi:10.1029/JA095iA08p11957

65. Lepri ST, Landi E, and Zurbuchen TH. Solar Wind Heavy Ions over Solar Cycle 23:Ace/swics Measurements. ApJ (2013) 768:94. doi:10.1088/0004-637X/768/ $1 / 94$

66. Ruffenach A, Lavraud B, Owens MJ, Sauvaud J-A, Savani NP, Rouillard AP, et al. Multispacecraft Observation of Magnetic Cloud Erosion by Magnetic Reconnection during Propagation. J Geophys Res (2012) 117:a. doi:10.1029/2012JA017624

67. Chen Y, Esser R, and Hu Y. Formation of Minor-Ion Charge States in the Fast Solar Wind: Roles of Differential Flow Speeds of Ions of the Same Element. ApJ (2003) 582:467-74. doi:10.1086/344642

68. Landi E, Gruesbeck JR, Lepri ST, Zurbuchen TH, and Fisk LA. Charge State Evolution in the Solar Wind. II. Plasma Charge State Composition in the Inner Corona and Accelerating Fast Solar Wind. ApJ (2012) 761:48. doi:10.1088/ 0004-637X/761/1/48

69. Buergi A, and Geiss J. Helium and Minor Ions in the Corona and Solar Wind Dynamics and Charge States. Solar Phys (1986) 103:347-83. doi:10.1007/ BF00147835

70. Boe B, Habbal S, Druckmüller M, Landi E, Kourkchi E, Ding A, et al. The First Empirical Determination of the Fe10+ and Fe13+ Freeze-In Distances in the Solar Corona. ApJ (2018) 859:155. doi:10.3847/1538-4357/aabfb7

71. Müller D, Marsden RG, Marsden RG, St. Cyr OC, and Gilbert HR. Solar Orbiter. Sol Phys (2013) 285:25-70. doi:10.1007/s11207-012-0085-7

72. Gan W, Yan Y, and Huang Y. Prospect for Space Solar Physics in 2016-2030. Scientia Sinica Physica, Mechanica \& Astronomica (2019) 49:059602. doi:10.1360/SSPMA2018-00301

73. Lin J, Wang M, Tian H, Song H, Fu H, and Huang M. In Situmeasurements of the Solar Eruption. Scientia Sinica Physica, Mechanica \& Astronomica (2019) 49:059607. doi:10.1360/SSPMA2018-00308

74. Wang Y, Ji H, Wang Y, Xia L, Shen C, Guo J, et al. Concept of the Solar Ring mission: An Overview. Sci China Technol Sci (2020) 63:1699-713. doi:10.1007/ s11431-020-1603-2

Conflict of Interest: The authors declare that the research was conducted in the absence of any commercial or financial relationships that could be construed as a potential conflict of interest.

Copyright $\odot 2021$ Song, Hu, Cheng, Zhang, Li, Zhao, Wang, Zheng and Chen. This is an open-access article distributed under the terms of the Creative Commons Attribution License (CC BY). The use, distribution or reproduction in other forums is permitted, provided the original author(s) and the copyright owner(s) are credited and that the original publication in this journal is cited, in accordance with accepted academic practice. No use, distribution or reproduction is permitted which does not comply with these terms. 